\title{
Effects of Resistance Exercise Applied Early After Coronary Artery Bypass Grafting: a Randomized Controlled Trial
}

Nayana Nazaré Pessoa Sousa Ximenes ${ }^{1,2}$, PT; Daniel Lago Borges', PT, MSc; Reijane Oliveira Lima1, PT; Mayara Gabrielle Barbosa e Silva', PT; Luan Nascimento da Silva', PT; Marina de Albuquerque Gonçalves Costa', PT; Thiago Eduardo Pereira Baldez', PT; Vinícius José da Silva Nina', MD, PhD

\section{Abstract}

Objective: To evaluate the effects of resistance exercise applied early after coronary artery bypass grafting.

Methods: It is a randomized controlled trial with $\mathbf{3 4}$ patients undergoing coronary artery bypass grafting between August 2013 and May 2014. Patients were randomized into two groups by simple draw: a control group $(n=17)$, who received conventional physical therapy and an intervention group $(n=17)$, who received, additionally, resistance exercise. Pulmonary function and functional capacity were evaluated in preoperative period and hospital discharge by spirometry and the six-minute walk test. For statistical analysis, we used the following tests: Shapiro-Wilk, Mann-Whitney, Student's $t$ and Fisher's exact. Variables with $P<0.05$ were considered significant.
Results: Groups were homogeneous in terms of demographic, clinical and surgical variables. Resistance exercise exerted no effect on pulmonary function of intervention group compared to control group. However, intervention group maintained functional capacity at hospital discharge measured by percentage of predict distance in 6 MWT $(54.1 \pm 22.7 \%$ vs. $52.5 \pm 15.5 \%, P=0.42)$, while control group had a significant decrease $(59.2 \pm 11.1 \%$ vs. $50.6 \pm 9.9 \%, P<0.016)$.

Conclusion: Our results indicate that resistance exercise, applied early, may promote maintenance of functional capacity on coronary artery bypass grafting patients, having no impact on pulmonary function when compared to conventional physical therapy.

Keywords: Exercise. Rehabilitation. Myocardial Revascularization.

\begin{tabular}{ll}
\hline Abbreviations, acronyms \& symbols \\
\hline 6MWT & $=$ Six-minute walk test \\
ACSM & $=$ American College of Sports Medicine \\
AHA & $=$ American Heart Association \\
BMI & $=$ Body mass index \\
CABG & $=$ Coronary artery bypass grafting \\
FEV1 & $=$ Forced expiratory volume in the first second \\
FEV1/FVC & $=$ Forced expiratory coefficient in the first second \\
FVC & $=$ Forced vital capacity \\
ICU & $=$ Intensive care unit \\
LL & $=$ Lower limbs \\
PEF & $=$ Peak expiratory flow \\
UL & $=$ Upper limbs
\end{tabular}

${ }^{1}$ Hospital Universitário da Universidade Federal do Maranhão (HUUFMA), São Luís, MA, Brazil.

${ }^{2}$ Programa de Pós-Graduação em Saúde do Adulto e da Criança, UFMA, São Luis, MA, Brazil.

This study was carried out at Hospital Universitário da Universidade Federal do Maranhão (HUUFMA), São Luís, MA, Brazil.

No financial support.

\section{INTRODUCTION}

Coronary artery bypass grafting (CABG) is the main treatment for coronary artery disease when medicines or percutaneous procedures are not enough to revert symptoms ${ }^{[1,2]}$. Over the past 10 years, the use of more durables grafts, aiming to minimize injuries to the patients, brought a progressive improvement to this surgical procedure ${ }^{[3]}$.

This progress has also encouraged advancements in the area of cardiac rehabilitation, which uses supervised exercise as therapeutic basis ${ }^{[4,5]}$. It is known that the practice of regular physical activity is beneficial and recommended as one of the best treatments for patients undergoing cardiac surgery, intending to reduce the risk of future cardiac events ${ }^{[6]}$.

Therefore, cardiac rehabilitation protocols have been developed to restore patients' daily activities, emphasizing physical and educational activities aiming lifestyle changes. Recent therapeutic techniques allow early hospital discharge with minimal reduction of functional capacity ${ }^{[5,7]}$.

Correspondence Address:

Daniel Lago Borges

Hospital Universitário da Universidade Federal do Maranhão (HUUFMA)

Rua Barão de Itapary, 227 - Centro - São Luis, MA, Brazil - Zip code: 65020-070 E-mail: dlagofisio83@hotmail.com

Article received on July $29^{\text {th }}, 2015$ Article accepted on November $3^{\text {rd }}, 2015$ 
In this respect, these patients have performed not only aerobic exercises but also resistance exercises and this practice is recommended for increase in functional capacity. The benefits of resistance exercise associated to aerobic exercise include an overall decrease of recurrent cardiac events, increased survival, physical and psychosocial independence, and improved quality of life ${ }^{[8]}$.

Since the first guidelines about resistance training established in 2000 by American Heart Association (AHA) and American College of Sports Medicine (ACSM), this exercise modality has become more accepted and included in exercise programs for subjects with and without cardiovascular disease. Brazilian Guidelines for Cardiac Rehabilitation and the ACSM report that muscle strength is fundamental to health, to the maintenance of functional capacity, and to achievement of satisfactory quality of life ${ }^{[5,9]}$.

Considering the above, our aim consists in evaluate the effects of resistance exercise applied early after coronary artery bypass grafting on functional capacity and pulmonary function.

\section{METHODS}

\section{Type and location of the study}

This randomized clinical trial was conducted in a Brazilian university hospital.

\section{Study sample}

A non-probabilistic sample of adult patients undergoing CABG between August 2013 and May 2014 were included in our study. Patients not included had chronic obstructive pulmonary disease, neurological sequelae, neuromuscular disease or submitted to associated, emergency or off-pump surgery. Perioperative deaths, surgical re-intervention and patients who remained on mechanical ventilation longer than 24 hours or noninvasive ventilation longer than four hours were excluded.

Total sample consisted of 34 patients randomized by simple draw into two groups: a control group with 17 patients undergoing conventional physical therapy and an intervention group with 17 patients undergoing resistance exercise (Figure 1).

\section{Patient evaluation}

In preoperative period, patients were informed and guided about the study and were subsequently evaluated. Data were collected from medical records and physical therapy evaluation form using a specific instrument developed for the study divided into three parts, containing data about pre-, intra-, and postoperative periods. Then, to assess pulmonary function, we performed spirometry, which involved the measurement forced vital capacity (FVC), forced expiratory volume in the first second (FEV1), forced expiratory coefficient in the first second (FEV1/ FVC [\%]), and peak expiratory flow (PEF), following criteria established by the guidelines for pulmonary function tests ${ }^{[10]}$. For mortality risk assessment we used EuroScore II $^{[11]}$.

The six-minute walk test (6MWT) was performed to evaluate functional capacity according to guidelines of American Thoracic Association ${ }^{[12]}$. The equation proposed by Iwama et al. ${ }^{[13]}$ was applied to estimate the predicted distance walked in the 6MWT. We performed spirometry and 6MWT preoperatively and at hospital discharge.

\section{Proposed treatments}

Patients included in control group received conventional physical therapy consisting of diaphragmatic breathing exercises and progressive ambulation, varying according to postoperative day and subjective tolerance to exercise assessed by Borg scale ${ }^{[14]}$. Additionally, were performed assisted and active exercises for upper (UL) and lower limbs (LL).

Intervention group received diaphragmatic breathing exercises, progressive ambulation and resistance exercises for UL and LL. Initial load was established using a stress test, which consisted of a set of 10 repetitions using $0.5 \mathrm{~kg}$ dumbbells for elbow flexion and $1.0 \mathrm{~kg}$ shin pads for knee extension. The load was defined by subjective perceived effort using Borg scale ${ }^{[6,14]}$.

Resistance exercises consisted of muscle training with dumbbells for UL (biceps and triceps) and shin pads for LL (quadriceps) with the patient in Fowler $45^{\circ}$, during immediate postoperative period, and subsequently sitting in bed with free LL allowing alternated and unilateral knee extension. Training

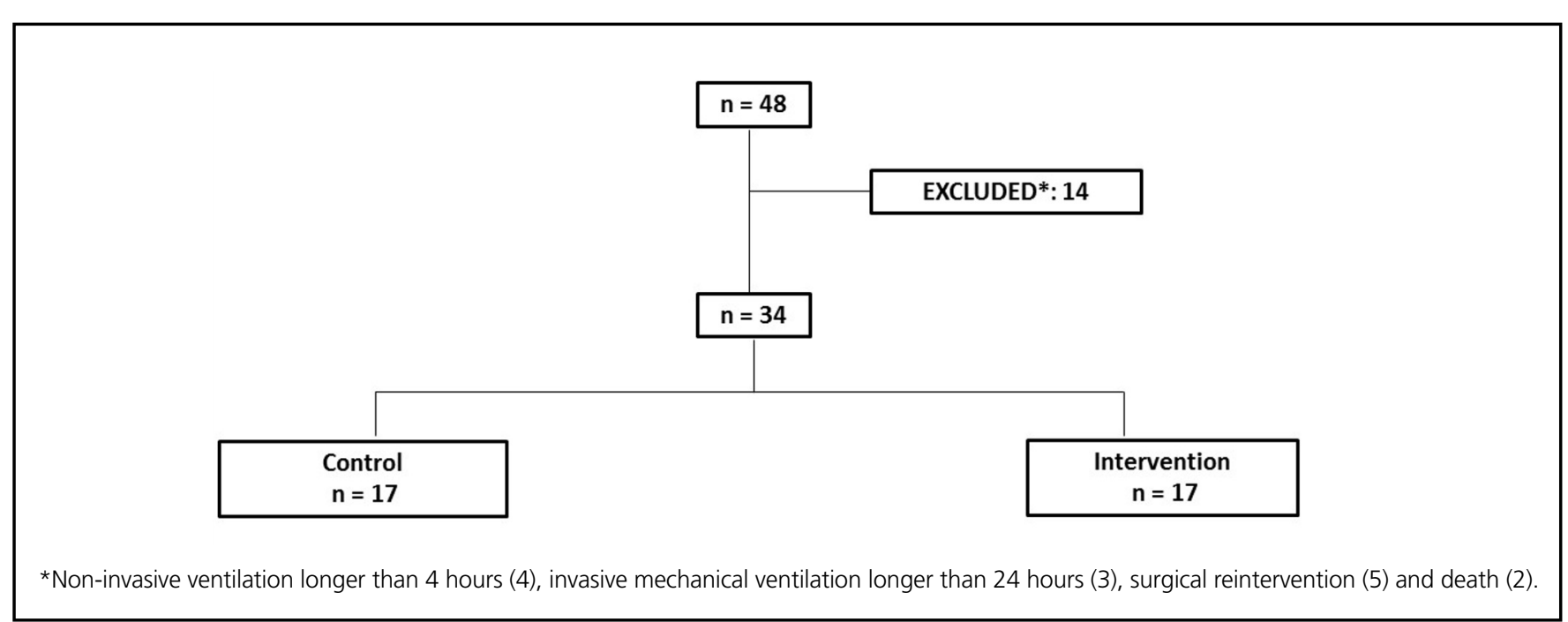

Fig. 1 - Flow chart. 
of hip adductors and abductors and triceps surae occurred at ward, where patient was free to ambulate, and consisted of three sets of 10 repetitions for each muscle group. Sessions were performed right after extubation in both the groups, for 30 minutes, twice a day in the intensive care unit (ICU) and once a day at ward until hospital discharge.

During exercises in the ICU, blood pressure, heart and respiratory rates, and oxygen saturation were monitored using a multiparameter monitor model Infinity Delta XL (Dräger Medical, Lübeck, Germany). At ward, before and after each session, blood pressure was measured using a digital sphygmomanometer (Panasonic model EW-BW30S, São Paulo, Brazil). Heart rate and oxygen saturation were monitored with a digital oximeter (Acc U Rate, United States)

\section{Statistical analysis}

Data collected were statistically analyzed using software Stata/SE 12.1 (Stata Corp, College Station, Texas, USA). To identify normality Shapiro-Wilk test was used. Quantitative variables were expressed as mean and standard deviation and their differences assessed using Mann-Whitney test for non-normal variables and Student's test for normal variables (independent and paired). For categorical variables, Fisher's exact test was used. The results were considered statistically significant when $P<0.05$.

\section{Ethical aspects}

In compliance with Resolution 466/12 of the National Health Council, the present study was approved by the Institutional Research Ethics Committee (No. 337.227).

\section{RESULTS}

In our study were included 48 patients. Fourteen were excluded due non-invasive ventilation longer than four hours $(n=4)$, mechanical ventilation longer than 24 hours $(n=3)$, surgical reintervention $(n=5)$ and death $(n=2)$ (Figure 1). Mortality rate in this population was $4.2 \%$.

Therefore, the final sample consisted of 34 patients, predominantly men (76.5\%), mean age of $60.9 \pm 6.8$ years, body mass index (BMI) of $25.7 \pm 3.3 \mathrm{~kg} / \mathrm{m}^{2}$, and EuroScore II of $0.76 \pm 0.14 \%$. Groups were homogeneous in terms of demographic, clinical, and surgical characteristics (Table 1).

Intubation time, length of stay in ICU and hospital were not significantly different between the groups, with an average duration of $14.2 \pm 7$ hours, $3.3 \pm 1$ days and $6.9 \pm 2.1$ days, respectively (Table 1).

Spirometry results assessed preoperatively and at hospital discharge are shown in Table 2. In both groups, there was a significant decrease in FVC, FEV1 and PEF at hospital discharge compared with the values obtained in preoperative period.

Table 1. Demographic, clinical, surgical and postoperative data, per group, of patients undergoing CABG.

\begin{tabular}{|c|c|c|c|c|}
\hline Variables & $\begin{array}{l}\text { Intervention } \\
(n=17)\end{array}$ & $\begin{array}{l}\text { Control } \\
(n=17)\end{array}$ & $\begin{array}{l}\text { Total } \\
(\%)\end{array}$ & $P$ \\
\hline $\begin{array}{l}\text { Gender } \\
\text { Male } \\
\text { Female }\end{array}$ & $\begin{array}{l}14 \\
3\end{array}$ & $\begin{array}{l}12 \\
5\end{array}$ & $\begin{array}{l}26(76.5) \\
8(23.5)\end{array}$ & $0.69^{a}$ \\
\hline Age (years) & $59.9 \pm 7$ & $61.8 \pm 6.7$ & $60.9 \pm 6.8$ & $0.43^{b}$ \\
\hline BMI $\left(\mathrm{kg} / \mathrm{m}^{2}\right)$ & $26.4 \pm 3.1$ & $24.9 \pm 3.3$ & $25.7 \pm 3.3$ & $0.17^{b}$ \\
\hline $\begin{array}{l}\text { Comorbidities } \\
\text { Hypertension } \\
\text { Smoke } \\
\text { Myocardial infarction } \\
\text { Diabetes mellitus } \\
\text { Dyslipidemia }\end{array}$ & $\begin{array}{l}13 \\
13 \\
10 \\
6 \\
8\end{array}$ & $\begin{array}{l}14 \\
9 \\
8 \\
8 \\
6\end{array}$ & 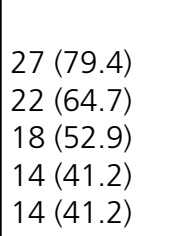 & $\begin{array}{l}1.00^{a} \\
0.28^{a} \\
0.52^{a} \\
0.73^{a} \\
0.73^{a}\end{array}$ \\
\hline EUROSCORE II (\%) & $0.73 \pm 0.18$ & $0.79 \pm 0.17$ & $0.76 \pm 0.14$ & $0.34^{b}$ \\
\hline Number of bypass graftings ( $n$ ) & $2.7 \pm 0.9$ & $2.7 \pm 0.4$ & $2.7 \pm 0.6$ & $0.68^{c}$ \\
\hline CPB time (min) & $84.2 \pm 35.9$ & $77.6 \pm 20.8$ & $80.2 \pm 28.2$ & $0.88^{c}$ \\
\hline Aortic clamp time (min) & $59.2 \pm 25$ & $55 \pm 13.7$ & $57.5 \pm 20.3$ & $0.54^{b}$ \\
\hline Surgery time (min) & $225.2 \pm 44.6$ & $243.1 \pm 60.3$ & $233.2 \pm 53.8$ & $0.30^{b}$ \\
\hline Intubation time (hours) & $14 \pm 7.8$ & $14.3 \pm 6.1$ & $14.2 \pm 7$ & $0.60^{c}$ \\
\hline ICU stay (days) & $3.2 \pm 1.2$ & $3.3 \pm 0.9$ & $3.3 \pm 1$ & $0.57^{c}$ \\
\hline Hospital stay (days) & $6.3 \pm 1.2$ & $7.5 \pm 2.7$ & $6.9 \pm 2.1$ & $0.10^{c}$ \\
\hline
\end{tabular}

$\mathrm{BMI}=$ body mass index; ICU=intensive care unit; ${ }^{2}$ Fisher's exact test. ${ }^{\mathrm{b}}$ Student's $\mathrm{t}$ test. 'Mann-Whitney test. Quantitatives variables showed as mean \pm standard deviation. 
Table 2. Pulmonary function at baseline and after early resistance exercise and control groups.

\begin{tabular}{|c|c|c|c|}
\hline & Intervention & Control & $P$ \\
\hline $\begin{array}{l}\text { FVC (\%) } \\
\text { Preoperative } \\
\text { Hospital discharge } \\
P\end{array}$ & $\begin{array}{l}77.7 \pm 13.3 \\
49.7 \pm 13.3 \\
<0.0001 \\
\end{array}$ & $\begin{array}{l}81.7 \pm 16.9 \\
58.3 \pm 13.9 \\
0.0004 \\
\end{array}$ & $\begin{array}{l}0.53 \\
0.09\end{array}$ \\
\hline $\begin{array}{l}\text { FEV1 (\%) } \\
\text { Preoperative } \\
\text { Hospital discharge } \\
P\end{array}$ & $\begin{array}{l}83.9 \pm 14.8 \\
54.2 \pm 14.8 \\
<0.0001 \\
\end{array}$ & $\begin{array}{l}89 \pm 15.3 \\
61.8 \pm 15.7 \\
0.0002 \\
\end{array}$ & $\begin{array}{l}0.65 \\
0.14\end{array}$ \\
\hline $\begin{array}{l}\text { FEV1/FVC (\%) } \\
\text { Preoperative } \\
\text { Hospital discharge } \\
P\end{array}$ & \begin{tabular}{|l|}
$108.8 \pm 8.3$ \\
$110.6 \pm 4.9$ \\
0.57 \\
\end{tabular} & $\begin{array}{l}107.2 \pm 12.9 \\
106.7 \pm 9.7 \\
0.53 \\
\end{array}$ & $\begin{array}{l}0.94 \\
0.13\end{array}$ \\
\hline $\begin{array}{l}\text { PEF (\%) } \\
\text { Preoperative } \\
\text { Hospital discharge } \\
P\end{array}$ & \begin{tabular}{|l|}
$64.9 \pm 18.5$ \\
$44.5 \pm 14.8$ \\
0.002 \\
\end{tabular} & $\begin{array}{l}60 \pm 22.2 \\
47.4 \pm 16.2 \\
0.04\end{array}$ & $\begin{array}{l}0.45 \\
0.29\end{array}$ \\
\hline
\end{tabular}

Intra-group analysis indicated that the control group subjected to conventional physical therapy showed a significant decrease $(P=0.016)$ in the predicted distance on the 6MWT when comparing preoperative and hospital discharge assessments. However, the intervention group submitted to resistance exercise maintained the predicted distance on the 6MWT (Table 3 and Figure 2).
Table 3. Intra and intergroups analysis of mean predicted distance of six minute walk test in patients undergoing CABG.

\begin{tabular}{l|l|l|l}
\hline & Preoperative & $\begin{array}{l}\text { Hospital } \\
\text { discharge }\end{array}$ & $\boldsymbol{P}$ \\
\hline Control (\%) & $59.2 \pm 11.1$ & $50.6 \pm 9.9$ & 0.016 \\
\hline Intervention (\%) & $54.1 \pm 22.7$ & $52.5 \pm 15.5$ & 0.42 \\
\hline $\boldsymbol{P}$ & 0.18 & 0.96 & \\
\hline
\end{tabular}

Data showed as mean \pm standard deviation. Mann-Whitney test.

\section{DISCUSSION}

Patients undergoing cardiac surgery may present clinical and functional disorders, highlighting pulmonary dysfunction, which leads to decreased lung volume and lung compliance, impaired respiratory function and increased respiratory effort. Decrease in lung volumes and capacities contributes to alterations in gas exchange, resulting in hypoxemia and decreased diffusion ${ }^{[15]}$.

A previous study that compared pulmonary outcomes in patients undergoing CABG indicated that on-pump surgery worsened lung compliance, gas exchange and prolonged intubation time ${ }^{[16]}$. In this regard, all patients in our study underwent on-pump surgery and, therefore, pulmonary dysfunction is expected.

Concerning to respiratory mechanics, our results showed a significant decrease in lung volume and capacity after $C A B G$, in both groups, as other studies in this population ${ }^{[15,17]}$. Oliveira et al. ${ }^{[18]}$ suggested that the decrease in FVC and FEV occurs by increased respiratory effort, shallower breathing due pain and decreased chest expansion secondary to sternotomy and surgical manipulation, resulting in restrictive ventilatory dysfunctions after surgery.

\section{Six-minute walk test}

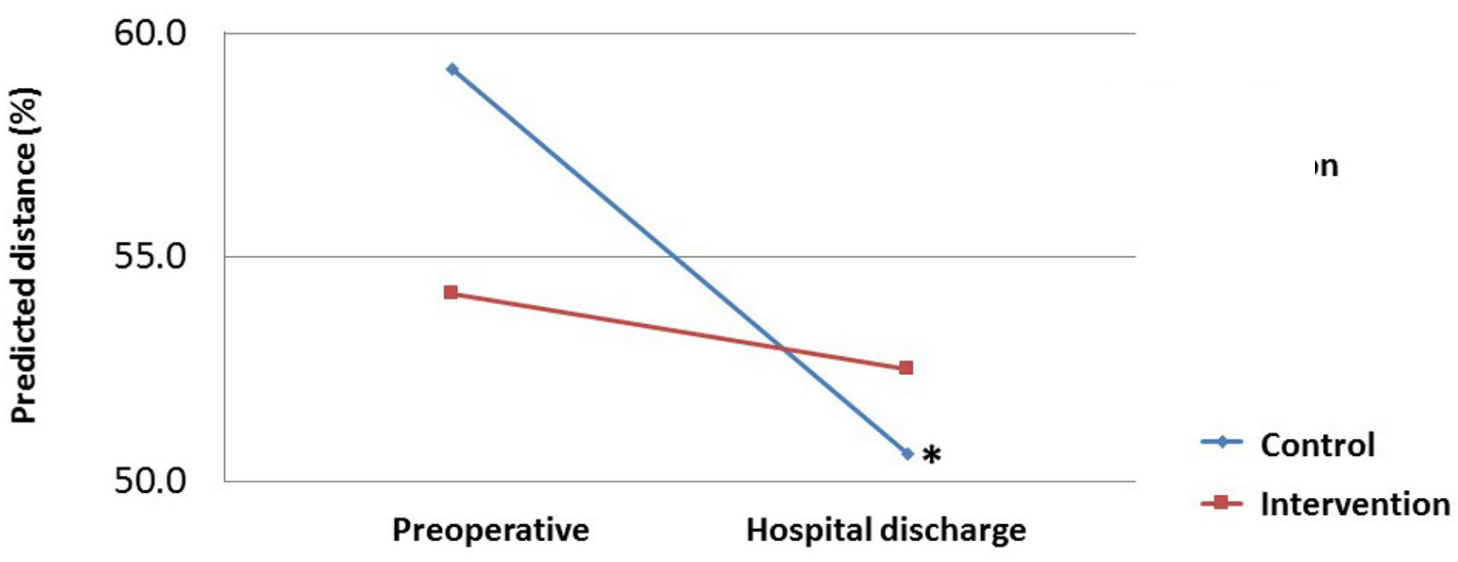

Fig. 2 - Functional capacity pre-exercise, and post-exercise in the intervention and control groups.

Mann-Whitney test.

${ }^{\star} P=0.016$ between preoperative and hospital discharge in control group. 
Decreased FVC and FEV after cardiac surgery affects coughing and sputum clearance, which may lead to obstruction of small airways, predispose the occurrence of micro-atelectasis and reduction of oxygenation, increasing hospital stay ${ }^{[19]}$.

Exercise programs are essentials for patients undergoing cardiac surgery ${ }^{[20]}$. El-Ansary et al. ${ }^{[21]}$ relate that, although pulmonary dysfunction is common in postoperative period, musculoskeletal disorders are underdiagnosed after cardiac surgery, with an incidence between $40 \%$ and $80 \%{ }^{[4]}$.

Recently, Gonçalves et al. ${ }^{[9]}$ reported that patients showed good tolerance during the practice of resistance exercise and it was considered safe due to lack of complications or cardiovascular events. These authors related as benefits of resistance exercise in cardiac patients: health improvement, control of risk factors and functional capacity increase. However, it is necessary to emphasize the importance of specific and individual evaluations prior to exercise prescription.

We found decrease in predicted distance walked in 6MWT between preoperative period and hospital discharge, as expected. However, patients who performed resistance exercise maintained their functional capacity when compared to control group.

Kawauchi et al. ${ }^{[20]}$ compared two physical therapy programs, involving a control group (routine protocol) and a training group, submitted to resistance exercise after cardiac transplantation, starting right after extubation. These authors observed similar results in both groups, with improvement in pulmonary function and functional capacity, concluding that the novel treatment program was as beneficial as routine program.

We evaluated the effects of resistance exercise applied early after $C A B G$ patients, starting the protocol in the first postoperative day. Researches about this topic, especially, concerning early resistance exercise in this population are scarce, which difficult comparisons of our results, emphasizing the importance of this study.

\section{Limitations}

Some patients presented pulmonary dysfunction and/or complications, requiring noninvasive or invasive ventilation for a long period or even surgical reintervention. Furthermore, peripheral muscle strength was not assessed.

\section{CONCLUSION}

Our results indicate that resistance exercise, applied early, may promote maintenance of functional capacity on CABG patients, having no impact on pulmonary function when compared to conventional physical therapy.

\section{Authors' roles \& responsibilities}

NNPSX Design and drawing of the study; implementation of projects and/or experiments; writing of the manuscript or critical review of its contents; final approval of the manuscript

DLB Design and drawing of the study; implementation of projects/experiments; analysis/interpretation of data; statistical analysis; manuscript writing or critical review of its contents; final approval of the manuscript

ROL Execution of operations/experiments

MGBS Execution of operations/experiments; manuscript writing or critical review of its contents; final approval of the manuscript

LNS Execution of operations/experiments; final approval of the manuscript

MAGC Execution of operations/experiments; final approval of the manuscript

TEPB Execution of operations/experiments; final approval of the manuscript

VJSN Design and drawing of the study; analysis/interpretation of data; writing of the manuscript writing or critical review of its contents; final approval of the manuscript

\section{REFERENCES}

1. Pêgo-Fernandes PM, Gaiotto FA, Guimarães-Fernandes F. Coronary artery bypass graft: state of the art. Rev Med (São Paulo). 2008;87(2):92-8

2. Montalescot G, Sechtem U, Achenbach S, Andreotti F, Arden C, Budaj A, et al. ESC Committee for Practice Guidelines. 2013 ESC guidelines on the management of stable coronary artery disease: the Task Force on the management of stable coronary artery disease of the European Society of Cardiology. Eur Heart J. 2013;34(38):29493003.

3. Windecker S, Kolh P, Alfonso F, Collet JP, Cremer J, Falk V, et al. 2014 ESC/EACTS Guidelines on myocardial revascularization. The Task Force on Myocardial Revascularization of the European Society of Cardiology (ESC) and the European Association for Cardio-Thoracic Surgery (EACTS) developed with the special contribution of the European Association of Percutaneous Cardiovascular Interventions (EAPCI). Eur Heart J. 2014;35(37):2541-619.

4. Viana PADC, Oliveira FTO, Esquivel MS, Tudella GO, Gardenghi G, Petto J. Exercise neuromuscular postoperative cardiac surgery. Rev DERC. 2014;20(1):18-21.

5. Moraes RS, Nóbrega $A C L$, Castro RRT, Negrão $C E$, Stein $R$, Serra SM, et al. Diretriz de reabilitação cardíaca. Arq Bras Cardiol. 2005;84(5):431-40.

6. Mellett LH, Bousquet G. Cardiology patient page. Heart-healthy exercise. Circulation. 2013;127(17):e571-2.

7. Machado MGR. Bases da fisioterapia respiratória: terapia intensiva e reabilitação. Rio de Janeiro: Guanabara Koogan; 2013.

8. Samayoa L, Grace SL, Gravely S, Scott LB, Marzolini S, Colella TJ. Sex differences in cardiac rehabilitation enrollment: a meta-analysis. Can J Cardiol. 2014;30(7):793-800.

9. Gonçalves ACCR, Pastre CM, Camargo JCS. Resistance exercise in heart disease: systematic review. Fisioter Mov. 2012;25(1):195-205.

10. Pereira CAC, Neder JA. Diretrizes para testes de função pulmonar. J Pneumol. 2002;28(supl. 3):1-238.

11. Lisboa LAF, Mejia OAV, Moreira LFP, Dallan LAO, Pomerantzeff PMA, Dallan LRP, et al. EuroSCORE II and the importance of a local 
model, InsCor and the future SP-SCORE. Braz J Cardiovasc Surg. 2014;29(1):1-8.

12. ATS statement: guideline for the six-minute walk test. Am J Respir Crit Care Med. 2002;166(1):111-7.

13. Iwama AM, Andrade GN, Shima P, Tanni SE, Godoy I, Dourado VZ. The six-minute walk test and body weight-walk distance product in healthy Brazilian subjects. Braz J Med Biol Res. 2009;42(11):1080-5.

14. Borg G. Psychophysical bases of perceived exertion. Med Sci Sports Exerc. 1982;14(5):377-81

15. Ferreira LL, Marino LHC, Cavenaghi S. Cardiopulmonary physical therapy in patients with heart disease. Rev Soc Bras Clin Med. 2012;10(2):127-31.

16. Guizilini S, Gomes WJ, Faresin SM, Bolzan DW, Alves FA, Catani R. Evaluation of pulmonary function in patients following on- and off-pump coronary artery bypass grafting. Braz J Cardiovasc Surg. 2005;20(3):310-6
17. Killewich LA. Strategies to minimize postoperative deconditioning in elderly surgical patients. J Am Coll Surg. 2006;203(5):735-45.

18. Oliveira EK, Silva VZM, Turquetto ALR. Relationship of postoperative walk test and lung function with the length of heart surgery hospital stay. Braz J Cardiovasc Surg 2009;24(4):478-84.

19. Leguisamo CP, Kalil RAK, Furlani AP. Effectiveness of a preoperative physiotherapeutic approach in myocardial revascularization. Braz J Cardiovasc Surg 2005;20(2):134-41.

20. Kawauchi TS, Almeida PO, Lucy KR, Bocchi EA, Feltrim MIZ, Nozawa E. Randomized and comparative study between two intra-hospital exercise programs for heart transplant patients. Braz J Cardiovasc Surg. 2013;28(3):338-46.

21. El-Ansary D, Adams R, Ghandi A. Musculoskeletal and neurological complications following coronary artery bypass graft surgery: a comparison between saphenous vein and internal mammary artery grafting. Aust J Physiother. 2000;46(1):19-25. 